\title{
Comparing Model Simulation and Experimental Results to Study the Dependence on Shear Stress of NO, ATP and ADP Production from Endothelial Cells
}

\author{
Dov Jaron, Patrick Kirby, Kenneth A. Barbee, Jaimit Parikh, and Donald G. Buerk \\ School of Biomedical Engineering, Science and Health Systems Drexel University Philadelphia, PA 19104
}

The concentrations of nitric oxide (NO) and adenine nucleotides (ATP, ADP) produced by cultured endothelial cells (ECs) in a parallel-plate flow chamber apparatus in response to changes in shear stress were compared to predictions generated by mathematical simulation. The model for NO production in a parallel flow chamber was based on earlier work, previously published by our group (1). Two models for adenine nucleotide production were used in the current study: a model developed by John and Barakat (2) and a model developed by Qin et al (3). The latter model was developed by fitting experimental results obtained by Yamamoto et al (4). The models were used to investigate the effect of changes in wall shear stress on production and transport of the three chemical species. In addition, experimental results using apyrase, which catalyses the hydrolysis of ATP to ADP and then to AMP, were compared to predictions of the simulations.

Comparisons of the mathematical simulations with the experimental observations indicate that a component of the EC's NO production is dependent upon the total nucleotide concentration at the EC surface. The inclusion of apyrase in both the experiments and in the mathematical model results in a decrease in the shear stress- dependent production of NO. The greatest effect of apyrase on the rate of NO production was observed at the lower range of shear stress considered in our studies.

The results of the simulation are useful in explaining experimental observations obtained using a parallel-plate chamber and expanding on the significant role of ATP autocrine stimulation in shear-dependent NO production by ECs.

\section{REFERENCES}

1. Fadel AA, Barbee KA, Jaron D. A computational model of nitric oxide production and transport in a parallel plate flow chamber. Annals of Biomedical Engineering. 2009;37(5):943-54.

2. John K, Barakat AI. Modulation of ATP/ADP concentration at the endothelial surface by shear stress: effect of flow-induced ATP release. Annals of Biomedical Engineering. 2001;29(9):740-51.

3. Qin K, Xiang C, Xu Z, Cao L, Ge S, Jiang Z. Dynamic modeling for shear stress induced ATP release from vascular endothelial cells. Biomechanics and Modeling in Mechanobiology. 2008;7(5):345-53.

4. Yamamoto K, Sokabe T, Ohura N, Nakatsuka H, Kamiya A, Ando J. Endogenously released ATP mediates shear stress-induced $\mathrm{Ca}^{2+}$ influx into pulmonary artery endothelial cells. American Journal of Physiology - Heart and Circulatory Physiology. 2003;285(2): H793-H803.

\footnotetext{
The original version of this chapter was inadvertently published with an incorrect chapter pagination 518 and DOI 10.1007/978-3-31932703-7_100. The page range and the DOI has been re-assigned. The correct page range is 524 and the DOI is 10.1007/978-3-31932703-7_101. The erratum to this chapter is available at DOI: 10.1007/978-3-319-32703-7 260
}

E. Kyriacou et al. (eds.), XIV Mediterranean Conference on Medical and Biological Engineering and Computing 2016, IFMBE Proceedings 57, 